\title{
RIQUEZA E ESTRUTURA DO COMPONENTE ARBÓREO E CARACTERÍSTICAS EDÁFICAS DE UM GRADIENTE DE FLORESTA CILIAR EM MINAS GERAIS, BRASIL ${ }^{1}$
}

\author{
Priscyla Maria Silva Rodrigues ${ }^{2}$, Sebastião Venâncio Martins ${ }^{3}$, Andreza Viana $\mathrm{Neri}^{4}$, Yule Roberta \\ Ferreira Nunes ${ }^{5}$ e Luiz Fernando Silva Magnago ${ }^{6}$
}

\begin{abstract}
RESUMO - Este estudo teve como objetivo relacionar as variações na riqueza e estrutura da comunidade arbórea com variáveis edáficas em um gradiente de floresta ciliar, no Parque Estadual da Mata Seca, Norte de Minas Gerais. Os estudos das variáveis edáficas e da diversidade e estrutura da vegetação arbórea (DAP $\geq 5 \mathrm{~cm}$ ) foram conduzidos em 39 parcelas de $400 \mathrm{~m}^{2}$, divididas equitativamente em três trechos previamente selecionados, sendo eles: São Francisco (menor teor de umidade), Meio (alagamento durante a maior parte do ano) e Lagoa da Prata (alagamento durante a estação chuvosa). A análise de solo superficial $(0$ a $20 \mathrm{~cm})$ evidenciou diferenças significativas entre os diferentes trechos florestais. Nos três ambientes foram amostrados 2.482 indivíduos, pertencentes a 36 espécies, 31 gêneros e 16 famílias botânicas. Houve diferença significativa nos diferentes trechos no índice de diversidade de Shannon, número de indivíduos, área basal e dominância absoluta. A distribuição diamétrica da comunidade apresentou grande número de indivíduos nas menores classes, decrescendo gradualmente. Vale salientar que a área basal e a densidade de indivíduos obtiveram correlações significativas com a maior parte das variáveis edáficas analisadas. Assim, a estrutura dos três trechos estudados está correlacionada com os fatores texturais, químicos e umidade do solo, ocasionando variações fitossociológicas nas comunidades estudadas.
\end{abstract}

Palavras-chave: Fitossociologia; Floresta inundável; Variações edáficas.

\section{RICHNESS AND STRUCTURE OF THE TREE COMPONENT AND SOIL CHARACTERISTICS OF A GRADIENT OF RIPARIAN FOREST IN MINAS GERAIS, BRAZIL}

\begin{abstract}
This study aimed to relate variations in richness and tree community structure to soil characteristics in a gradient of riparian forest, at Parque Estadual da Mata Seca, north of Minas Gerais. Studies of soil characteristics and the richness and structure of trees $(D B H \geq 5 \mathrm{~cm})$ were conducted in 39 plots of $400 \mathrm{~m}^{2}$, divided equally into three sections previously selected, these being: São Francisco (lower moisture), Meio (flooding during most of the year) and Lagoa da Prata (flooding during the rainy season). The analysis of topsoill $(0-20 \mathrm{~cm})$ showed significant differences between the different forest sections. In all three environments were sampled 2482 individuals belonging to 36 species, 31 genera and 16 plant families. There were significant differences for the different sections in the Shannon diversity index, number of individuals, basal area and absolute dominance. The diameter distribution of the community had a great number of individuals concentred in the smallest classes, decreasing gradually. It is noteworthy that the basal area and density of subjects
\end{abstract}

\footnotetext{
${ }^{1}$ Recebido em 08.11.2012 aceito para publicação em 06.11.2013.

${ }^{2}$ Programa de Pós-graduação em Botânica, Universidade Federal de Viçosa, UFV, Brasil. E-mail:<priscylamsr@gmail.com>.

${ }^{3}$ Departamento de Engenharia Florestal, Universidade Federal de Viçosa, UFV, Brasil. E-mail:<venancio@ufv.br>.

${ }^{4}$ Departamento de Biologia Vegetal, Universidade Federal de Viçosa, UFV, Brasil. E-mail:<andrezaneri@gmail.com>.

${ }^{5}$ Programa de Pós-Graduação em Ciências Biológicas da Universidade Estadual de Montes Claros, UNIMONTES. E-mail:<yule.rfnunes@gmail.com>.

${ }^{6}$ Programa de Pós-graduação na Universidade Federal de Viçosa, Laboratório de ecologia e restauração florestal. E-mail:<luiz_fsm@hotmail.com>.
} 
had significant correlations with soil most variables analyzed. Thus, the structure of the three sections studied is correlated with the textural, chemical and soil moisture, causing variations in phytosociological communities studied.

Keywords: Phytosociology; Flooded riparian forest; Edaphic variations.

\section{INTRODUÇÃO}

A florística e a estrutura da vegetação mostram-se fortemente influenciadas pela natureza do substrato adjacente, principalmente os solos que sustentam as formações vegetacionais e são responsáveis pela quantidade de nutrientes e água fornecidos às espécies que sobre eles se desenvolvem (MARTINS et al., 2003; PINTO et al., 2008; SCHAEFER et al., 2009). Assim, vegetação e solo são extremamente relacionados, o que torna difícil identificar a causae-efeito (RUGGIERO et al., 2002). Fragmentos de florestas ciliares geralmente possuem variações edafoclimáticas relacionadas à distribuição e riqueza das espécies vegetais (HUSTON, 1980; BAILLIE; ASHTON, 1983), nitidamente perceptível no gradiente florestal. Entretanto, outros autores relacionaram essa variação a eventos estocásticos (WHITMORE, 1974; HUBBELL; FOSTER, 1983).

As formações ciliares possuem alta heterogeneidade florística, resultante da ação de fatores físicos e biológicos que atuam sobre elas, modificando as características do ambiente e regulando a distribuição e organização do mosaico vegetacional (RODRIGUES; NAVE, 2000). Essas florestas desempenham importante função ambiental e funcionam como filtro, retendo poluentes e agrotóxicos que seriam carreados para os cursos d’água. São, portanto, fundamentais para a manutenção da qualidade da água nas bacias hidrográficas (MARTINS, 2007).

Nessas áreas ciliares são encontrados diversos tipos de solos, que variam em função do maior ou do menor grau de hidromorfismo ou da ausência desse aspecto (JACOMINE, 2004). Diferentes períodos de inundação podem ocorrer nas florestas ciliares, o que reflete diretamente na fisionomia, composição e estrutura das comunidades vegetais (BIANCHINI et al., 2003). As inundações periódicas e de curta duração podem contribuir para a absorção de nutrientes que aumentam a produtividade e riqueza de espécies vegetais (DÉSILETS; HOULE, 2005). Em contraste, inundações de longa duração representam grande estresse e podem resultar em comunidades pobres em espécies de plantas, diminuindo, portanto, a produtividade e a riqueza (WITTMANN et al., 2004; DÉSILETS; HOULE, 2005; CAMARGOS et al., 2008).

Estudos fitossociológicos sobre florestas inundáveis no Norte de Minas Gerais são inexistentes (SILVA et al., 2007). Devido à escassez de informações nessas áreas, várias regiões do Norte e Noroeste de Minas Gerais são apontadas como prioritárias para investigação científica (DRUMMOND et al., 2005). Diante disso, é de extrema importância estudos nessas florestas, visando conhecer a estrutura, bem como os fatores ecológicos determinantes da distribuição das espécies e diferenciação da vegetação. Essas informações são fundamentais para a conservação desses ecossistemas peculiares e para a definição de estratégias para restauração em áreas já degradadas.

Devido à importância da vegetação do rio São Francisco, este estudo teve como objetivo relacionar as variações na riqueza e estrutura da comunidade com as variáveis edáficas em gradiente de inundação em florestas ciliares no Parque Estadual da Mata Seca, Norte de Minas Gerais. Buscou-se responder às seguintes questões: (i) a riqueza e estrutura do componente arbóreo variam no gradiente amostrado?; (ii) como as variáveis ambientais testadas influenciam na estrutura e composição dessa comunidade? As hipóteses foram: (i) por se tratar de floresta que sofre com pulsos de inundação, a floresta ciliar do Parque Estadual da Mata Seca (PEMS) possui menor riqueza de espécies; (ii) ambientes que apresentam maior umidade no solo e menor disponibilidade nutricional possuem menor diversidade e maior dominância ecológica.

\section{MATERIAL E MÉTODOS}

\section{1. Área de estudo}

A área de estudo está localizada no PEMS, no Vale do Médio São Francisco, no Município de Manga, Minas Gerais. O PEMS possui uma área de 10.281,44 ha. A vegetação predominante é a Floresta Estacional Decidual, alterada em diferentes estágios sucessionais e pastagens (MADEIRA et al., 2009). As florestas ciliares 
variam de perenifólias a semideciduais, em que alguns elementos florísticos decíduos são abundantes (IEF, 2000). Essas florestas se encontram ao longo das lagoas marginais e do rio São Francisco. As florestas ciliares inseridas dentro do parque, mesmo protegidas pelo Código Florestal (Lei n ${ }^{4} .771 / 65$ ), vêm sofrendo degradação acelerada, devido à ocorrência de diversas práticas de uso antrópico, principalmente a agricultura de vazante.

Segundo a classificação de Köppen (1948), o clima predominante na região é o Aw, caracterizado pela existência das estações seca e chuvosa bem definidas. A temperatura média anual é de $24{ }^{\circ} \mathrm{C}$ e altitude de 400-500 m, sendo a precipitação média anual de 818 mm/ano (MADEIRA et al., 2009). Os solos predominantes nessa região são os Latossolos Vermelho-Amarelos, Neossolos Flúvicos e Cambissolos háplicos (MELLO et al., 2008; MADEIRA et al., 2009).

Os trechos florestais estudados apresentam-se de forma contígua, situados em um fragmento isolado de floresta ciliar inundável, que se encontra em gradiente entre o rio São Francisco e a lagoa da Prata, inserido entre as coordenadas 1449’26.51"S e 4355’37.06"W. $\mathrm{O}$ trecho às margens do rio São Francisco possui menor tempo de inundação durante o ano, enquanto o trecho às margens da lagoa da Prata apresenta alagamento durante a estação chuvosa. O trecho entre o rio e a lagoa aparece nas depressões sujeitas à inundação durante a maior parte do ano, sendo, portanto, o trecho com maior encharcamento denominado neste estudo como Meio.

\subsection{Amostragem da vegetação}

A amostragem do estrato arbóreo foi realizada utilizando-se o método de parcelas (MUELLERDOMBOIS; ELLENBERG, 1975). Foram alocadas 39 parcelas de $20 \mathrm{~m} \times 20 \mathrm{~m}\left(400 \mathrm{~m}^{2}\right)$, distando $10 \mathrm{~m}$ entre si, totalizando uma área amostral de 1,56 ha. As parcelas foram divididas equitativamente em três blocos, sendo um às margens do rio São Francisco, outro às margens da lagoa da Prata e o último localizado entre os dois primeiros blocos, denominado neste estudo como Meio.

Nas parcelas, todos os indivíduos arbóreos com Circunferência à Altura do Peito (CAP) a 1,30 m do solo $\geq 15,7 \mathrm{~cm}$ foram inventariados. Dessa forma, todos os indivíduos inventariados foram marcados com plaquetas de alumínio numeradas, sendo registrado o nome da espécie, quando identificado, e coletado material botânico (vegetativo e, ou, reprodutivo), além do CAP e da altura, esta estimada por observações visuais.

O material botânico coletado foi tratado segundo as técnicas convencionais de herborização e depositado, quando encontrado com material fértil, no Herbário VIC da Universidade Federal de Viçosa. As espécies foram classificadas nas famílias reconhecidas pelo sistema do Angiosperm Phylogeny Group III (APG III, 2009).

\subsection{Variáveis edáficas}

Para caracterizar o solo das áreas amostrais, em cada parcela de $400 \mathrm{~m}^{2}$ foram coletadas amostras compostas superficiais de solo $(0-20 \mathrm{~cm}$ de profundidade), totalizando 39 amostras. Cada amostra foi constituída de cinco subamostras, sendo uma coletada no centro da parcela e as outras quatro, nos seus vértices. As análises químicas, texturais e de umidade com base em massa foram realizadas no Laboratório do Departamento de Solos da Universidade Federal de Viçosa, obtendo-se as seguintes variáveis: $\mathrm{pH}$; teores de P, K, Ca, Mg, Al, Zn, Fe, Mn, Cu, B e S; H + Al; soma de bases trocáveis (SB); capacidade de troca catiônica efetiva (t); capacidade de troca catiônica a pH 7,0 (T); matéria orgânica; saturação por bases (V); e teores de areia grossa, areia fina, silte e argila e umidade do solo. Vale salientar que todas as análises seguiram os procedimentos da Embrapa(1997).

\subsection{Análise dos dados}

A estrutura do componente arbóreo foi descrita a partir do cálculo dos parâmetros quantitativos de frequência, densidade e dominância absolutas e relativas, bem como área basal e valor de importância (MUELLERDOMBOIS; ELLENBERG, 1975). As análises fitossociológicas foram realizadas no Programa Mata Nativa 2 (CIENTEC, 2006).

Foram preparadas distribuições de densidade de árvores por classes de diâmetro e altura para os trechos amostrados. As classes de distribuição de diâmetro foram definidas de forma a se ajustarem ao número de indivíduos encontrado e à amplitude da variação do diâmetro na área, conforme a seguir: Classe 1, > 5 a $10 \mathrm{~cm}$; Classe 2, > 10 a $20 \mathrm{~cm}$; Classe 3, > 20 a 40 $\mathrm{cm}$; e Classe $4,>40$ a $80 \mathrm{~cm}$. Os intervalos de classe com amplitude exponencial crescente são adotados para compensar o declínio da densidade nas classes de tamanhos maiores e típico da distribuição exponencial

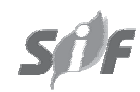

Revista Árvore, Viçosa-MG, v.37, n.6, p.1011-1023, 2013 
negativa (J-invertido), o que resulta em boa estrutura gráfica (OLIVEIRA-FILHO et al., 2001). No caso das alturas, a distribuição dos indivíduos foi baseada na altura normalmente alcançada pelas árvores, sendo definidas quatro classes: Classe $1,<4 \mathrm{~m}$; Classe 2, $\geq 4<8 \mathrm{~m}$; Classe $3, \geq 8<12 \mathrm{~m}$; e Classe $4, \geq 12 \mathrm{~m}$.

Os índices de diversidade de Shannon-Wiener (H'), equabilidade de Pielou (J') e índice de dominância de Simpson (C) foram calculados conforme Brower e Zar (1984), no Programa Mata Nativa 2 (CIENTEC, 2006). Para verificar as diferenças entre os índices de diversidade $H$ ' encontrados em cada trecho estudado no gradiente, foi empregado o teste $\mathrm{T}$ modificado (MAGURRAN, 1988).

Para comparar as parcelas quanto à similaridade florística, foi construída uma matriz de dados binários (presença e ausência) das espécies. A partir dessa matriz foi produzida uma matriz de coeficientes de similaridade de Jaccard. Para interpretar as similaridades florísticas entre as parcelas, empregou-se uma análise de agrupamento (UPGMA) (VALENTIM, 2000). As análises foram realizadas com o Programa PC-Ord versão 5.1 (MCCUNE; MEFFORD, 2006).

Foram realizadas análises de variância (one-way ANOVA), seguidas de testes de Tukey-Kramer, para verificar em quais grupos houve diferença significativa nas variáveis químicas, texturais e na umidade do solo (ZAR, 1998). As variáveis que expressam proporções (p) foram transformadas previamente pela função arcosseno $\mathrm{p}^{1 / 2}$. Para realização dessas análises, a normalidade das variáveis foi verificada tanto graficamente quanto pelo teste de Kolmogorov-Smirnov (Lilliefors) (ZAR, 1998). Esse mesmo procedimento foi utilizado para os parâmetros estruturais (número total de indivíduos, riqueza, área basal e dominância) das formações analisadas. As análises foram feitas utilizando-se o software Statistica 7.0 (STATSOFT, 2004).

Para avaliar a correlação das variáveis edáficas com a densidade e a área basal, foi calculado o Coeficiente de Correlação de Pearson (GILBERT, 1976). As variáveis pedológicas utilizadas para esse teste foram: $\mathrm{pH}$ em $\mathrm{H}_{2} \mathrm{O}$, fósforo $(\mathrm{P})$, potássio $(\mathrm{K})$, cálcio $(\mathrm{Ca})$, magnésio (Mg), alumínio (Al), MO (matéria orgânica), zinco (Zn), ferro (Fe), Mn (manganês), cobre (Cu), areia grossa, areia fina, silte, argila e umidade. As análises foram geradas através do software Statistica 7.0 (STATSOFT, 2004).

Revista Árvore, Viçosa-MG, v.37, n.6, p.1011-1023, 2013

\section{RESULTADOS}

\subsection{Variáveis edáficas}

Os três trechos da floresta ciliar estudados apresentaram diferenças significativas entre si para 15 das 16 variáveis edáficas comparadas (Tabela 1), o que representa um gradiente de características químicas e físicas. Com relação à classe textural, o solo do trecho São Francisco foi identificado como franco-arenoso para a maioria das parcelas amostradas (69\%). O trecho Meio compreendeu o grupo da argilo-siltosa, com exceção de uma parcela, onde o solo foi classificado como franco-argilo-siltoso. O trecho Lagoa da Prata possui 85\% das suas parcelas classificadas como argilosa.

O solo do São Francisco apresentou umidade, teores de Al, matéria orgânica, Fe e Cu e proporções de argila significativamente mais baixos que os solos do Meio e da Lagoa da Prata. Os teores Mg e Ca foram significativamente mais altos na Lagoa da Prata e mais baixos no São Francisco, sendo intermediários no Meio. O São Francisco obteve teores de pH, P, Zn e Mn significativamente mais altos que nos trechos Meio e lagoa da Prata. Os teores de K e proporções de areia fina foram maiores no São Francisco, intermediários no Meio e mais baixos na Lagoa da Prata. Já os teores de silte foram maiores no Meio, menores no São Francisco e intermediários na Lagoa da Prata. Não houve diferença significativa entre os três trechos para a proporção de areia grossa.

O solo do trecho São Francisco é considerado moderadamente drenado em relação aos solos dos outros dois trechos, apresentando maior porcentagem de areia e menor de argila e silte no perfil superficial. Já os solos do Meio e da Lagoa Prata possuem maiores teores de argila e silte no perfil superficial, sendo, por isso, menos lixiviáveis, no entanto têm maiores teores de Al, Fe e umidade. Não obstante, o São Francisco obteve alto teor de $\mathrm{Mn}$.

\subsection{Variações na diversidade e estrutura das comunidades}

Foram amostrados 2.482 indivíduos, que se distribuíram em 36 espécies, 31 gêneros e 16 famílias botânicas (Tabela 2). A diversidade indicada pelo índice de Shannon (H’) e a dominância ecológica, representada pelo índice de equabilidade ( $\mathrm{J}$ '), obtiveram valores de: $H^{\prime}=2,04$ e J'= 0,57. Além disso, o índice de dominância de Simpson foi de 0,80 . O valor de H' foi maior no trecho

Revista Árvore, Viçosa-MG, v.37, n.6, p.1011-1023, 2013 
Tabela 1 - Variáveis químicas, texturais e umidade de solo superficial (0-20 cm de profundidade) em gradiente de floresta ciliar inundável no PEMS, Norte de Minas Gerais. Análises de variância indicaram diferenças significativas entre os solos, e as médias seguidas de letras diferentes são significativamente diferentes pelo teste de Tukey $(\mathrm{p}<0,05)$

Table 1 - Chemical, textural and moisture variables of surface soil profile $(0-20 \mathrm{~cm})$ in a gradient of riparian wetland in the PEMS, north of Minas Gerais. Analysis of variance indicated significant differences among soils, and the means followed by different letters are significantly different in Tukey's tests

\begin{tabular}{|c|c|c|c|c|}
\hline Variáveis & São Francisco & Meio & Lagoa da Prata & $\operatorname{ANOVA}(\mathrm{F})$ \\
\hline Umidade (kg/kg) & $0,11 \pm 0,05 b$ & $0,17 \pm 0,02 a$ & $0,16 \pm 0,02 \mathrm{a}$ & $15,16 * * *$ \\
\hline $\mathrm{pH}$ em $\mathrm{H}_{2} \mathrm{O}$ & $7,0 \pm 0,3 a$ & $5,3 \pm 0,2 b$ & $5,3 \pm 0,1 b$ & $241,15 * * *$ \\
\hline $\mathrm{P}\left(\mathrm{mg} \cdot \mathrm{dm}^{-3}\right)$ & $15,33 \pm 2,64 a$ & $4,96 \pm 2,44 b$ & $5,23 \pm 1,66 b$ & $86,57 * * *$ \\
\hline $\mathrm{K}^{+}\left(\mathrm{mg} \cdot \mathrm{dm}^{-3}\right)$ & $107,23 \pm 17,87 a$ & $85,92 \pm 36,94 \mathrm{ab}$ & $79,08 \pm 17,44 b$ & $4,23 *$ \\
\hline $\mathrm{Ca}^{++}\left(\mathrm{mg} \cdot \mathrm{dm}^{-3}\right)$ & $5,85 \pm 1,23 b$ & $6,51 \pm 0,62 \mathrm{ab}$ & $7,19 \pm 0,61 \mathrm{a}$ & $7,75^{* *}$ \\
\hline $\mathrm{Mg}^{++}\left(\mathrm{mg} \cdot \mathrm{dm}^{-3}\right)$ & $0,92 \pm 0,14 \mathrm{c}$ & $1,55 \pm 0,15 b$ & $1,79 \pm 0,24 a$ & $79,35 * * *$ \\
\hline $\mathrm{Al}^{+++}\left(\mathrm{mg} \cdot \mathrm{dm}^{-3}\right)$ & $0,00 \pm 0,00 b$ & $0,23 \pm 0,16 a$ & $0,20 \pm 0,22 a$ & $7,72 * *$ \\
\hline Mat. orgânica (dag.kg-1) & $2,26 \pm 0,30 a$ & $3,59 \pm 0,54 b$ & $3,59 \pm 0,52 b$ & $28,30 * * *$ \\
\hline $\mathrm{Zn}\left(\mathrm{mg} \cdot \mathrm{dm}^{-3}\right)$ & $4,58 \pm 0,73 a$ & $2,42 \pm 0,71 b$ & $1,94 \pm 0,21 b$ & $70,26 * * *$ \\
\hline $\mathrm{Fe}\left(\mathrm{mg} \cdot \mathrm{dm}^{-3}\right)$ & $154,56 \pm 72,46 b$ & $219,97 \pm 48,11 \mathrm{a}$ & $213,80 \pm 44,27 a$ & $5,34 * *$ \\
\hline $\operatorname{Mn}\left(\mathrm{mg} \cdot \mathrm{dm}^{-3}\right)$ & $49,00 \pm 18,74 a$ & $15,48 \pm 3,97 b$ & $14,15 \pm 4,71 b$ & $39,09 * * *$ \\
\hline $\mathrm{Cu}\left(\mathrm{mg} . \mathrm{dm}^{-3}\right)$ & $0,52 \pm 0,37 b$ & $0,90 \pm 0,13 a$ & $1,01 \pm 0,57^{\mathrm{a}}$ & $5,44 * *$ \\
\hline Areia grossa (\%) & $0,69 \pm 0,48$ & $0,61 \pm 0,51$ & $0,46 \pm 0,51$ & $0,50 \mathrm{~ns}$ \\
\hline Areia fina (\%) & $61,77 \pm 16,43 a$ & $6,38 \pm 0,96 b$ & $21,00 \pm 4,77 c$ & $25,35 * * *$ \\
\hline Silte (\%) & $23,23 \pm 10,26 a$ & $46,69 \pm 2,84 b$ & $33,92 \pm 3,73 c$ & $42,31 * * *$ \\
\hline Argila (\%) & $14,31 \pm 6,34 a$ & $46,31 \pm 3,50 b$ & $44,61 \pm 2,36 b$ & $217,83^{* * *}$ \\
\hline
\end{tabular}

$* \mathrm{p}<0,05 ; * * \mathrm{p}<0,01 ; * * * \mathrm{p}<0,001 ;$ e ns = não significativo.

São Francisco $(1,92)$, seguido pelos trechos Lagoa da Prata (1,79) e Meio (1,69). O teste T de Magurran (1998) apontou que a diversidade do trecho Meio foi significativamente diferente dos trechos São Francisco e Lagoa da Prata. A baixa equabilidade encontrada nos três trechos demonstra que houve forte concentração de abundância em poucas espécies.

A análise de agrupamento das espécies no gradiente de floresta ciliar mostrou a formação de dois grupos florísticos bem distintos (Figura 1). Houve semelhança no nível de $10 \%$ no primeiro grupo (A), estabelecido nas parcelas localizadas no trecho São Francisco (113), enquanto o segundo grupo (B), no nível de similaridade de $45 \%$, consistiu em parcelas localizadas nos trechos Meio e Lagoa da Prata (27-39 e 14-26, respectivamente). O grupo Aé caracterizado por possuir o solo com textura mais arenosa e maior fertilidade, seguido pelo grupo B, o qual apresenta solo com textura mais argilosa, menor fertilidade e maior umidade.

O número de indivíduos amostrados diferiu entre os trechos ( $\mathrm{gl}=2 ; \mathrm{F}=21,999 ; \mathrm{p}<0,001)$, e o trecho Lagoa da Prata apresentou a maior densidade de indivíduos (1.180), seguida pelo Meio (909) e pelo São
Francisco (393). No entanto, o número de espécies amostradas não diferiu entre os trechos ( $\mathrm{gl}=2 ; \mathrm{F}=$ 1,706; p > 0,05), mostrando homogeneidade da floresta para esse parâmetro. O total de indivíduos inventariados na amostra somou uma área basal de $21,64 \mathrm{~m}^{2}$, e os resultados variaram significativamente entre os três trechos estudados ( $\mathrm{gl}=2 ; \mathrm{F}=7,654 ; \mathrm{p}<0,01)$. O São Francisco obteve o maior valor ( $\bar{X}=7,33 \pm 0,16 \mathrm{DP})$, seguido pela Lagoa da Prata ( $=5,70 \pm 0,38 \mathrm{DP})$ e pelo Meio ( = 3,58 \pm 0,11 DP).

A distribuição da densidade dos indivíduos por classe de altura (Figura 2A) mostrou que a classe $\geq$ $4<8$ m obteve menor densidade no trecho São Francisco que no Meio e na Lagoa da Prata, enquanto na classe de $\geq 8<12$ m de altura a densidade de árvores foi maior no São Francisco que nos outros dois trechos. A curva da distribuição de diâmetros dos indivíduos apresentou tendência exponencial negativa (“J invertido”) nos trechos Meio e Lagoa da Prata (Figura 2B). Portanto, as classes com maiores diâmetros obtiveram menor número de indivíduos. Com relação ao trecho São Francisco, a distribuição de indivíduos por classe diamétrica foi quase contínua. De forma geral, o São Francisco apresentou maior densidade de árvores mais 
Tabela 2 - Parâmetros fitossociológicos das espécies amostradas nos trechos "São Francisco, Meio e Lagoa da Prata", no PEMS, Norte de Minas Gerais, apresentados em ordem decrescente de VI (\%). NI = número de indivíduos em 0,52 ha; $\mathrm{AB}=$ área basal $\left(\mathrm{m}^{2}\right) ; \mathrm{DR}$ = densidade relativa; $\mathrm{FR}$ = frequência relativa; DoR = dominância relativa; e VI = valor de importância.

Table 2 - Phytossociological parameters of the surveyed species in the section "São Francisco, Meio e Lagoa da Prata", in the PEMS, north of Minas Gerais, presented in decreasing order of VI(\%). NI = number of individuals in 0,52 ha; $A B=$ basal area $\left(m^{2}\right) ; D R=$ relative density; $F R=$ relative frequency; Do $R=$ relative dominance; and $V I$ = importance value.

\begin{tabular}{|c|c|c|c|c|c|c|}
\hline Nome Científico/Trecho São Francisco & NI & $\mathrm{AB}$ & DR & FR & DoR & VI \\
\hline Celtis ehrenbergiana (Klotzsch) Liebm. & 211 & 5,29 & 53,69 & 13,13 & 55,44 & 40,75 \\
\hline Inga vera Willd. & 37 & 1,49 & 9,41 & 12,12 & 15,65 & 12,39 \\
\hline Ziziphus joazeiro Mart. & 17 & 0,67 & 4,33 & 6,06 & 7,00 & 5,80 \\
\hline Maclura tinctoria (L.) D.Don ex Steud. & 17 & 0,38 & 4,33 & 7,07 & 4,01 & 5,14 \\
\hline Albizia inundata (Mart.) Barneby \& J.W.Grimes & 14 & 0,23 & 3,56 & 9,09 & 2,46 & 5,04 \\
\hline Triplaris gardneriana Wedd. & 18 & 0,42 & 4,58 & 5,05 & 4,36 & 4,66 \\
\hline Anadenanthera colubrina (Vell.) Brenan & 11 & 0,33 & 2,80 & 7,07 & 3,46 & 4,44 \\
\hline Savia dictyocarpa. Müll.Arg. & 12 & 0,07 & 3,05 & 6,06 & 0,69 & 3,27 \\
\hline Chloroleucon dumosum (Benth.) G.P.Lewis & 8 & 0,09 & 2,04 & 5,05 & 0,97 & 2,68 \\
\hline Talisia esculenta (Cambess.) Radlk. & 7 & 0,06 & 1,78 & 5,05 & 0,62 & 2,48 \\
\hline Colubrina glandulosa Perkins & 12 & 0,05 & 3,05 & 3,03 & 0,50 & 2,19 \\
\hline Trichilia hirta L. & 4 & 0,03 & 1,02 & 4,04 & 0,26 & 1,77 \\
\hline Chloroleucon foliolosum (Benth.) G.P.Lewis & 4 & 0,02 & 1,02 & 3,03 & 0,17 & 1,41 \\
\hline Senna rizzinii H.S.Irwin \& Barneby & 2 & 0,13 & 0,51 & 2,02 & 1,36 & 1,30 \\
\hline Guarea guidonia (L.) Sleumer & 7 & 0,09 & 1,78 & 1,01 & 0,97 & 1,25 \\
\hline Senna spectabilis (DC.) H.S.Irwin \& Barneby & 2 & 0,02 & 0,51 & 2,02 & 0,19 & 0,90 \\
\hline Senegalia polyphylla (DC.) Britton \& Rose & 2 & 0,01 & 0,51 & 2,02 & 0,13 & 0,89 \\
\hline Piper arboreum Aubl. & 1 & 0,07 & 0,25 & 1,01 & 0,71 & 0,66 \\
\hline Pterogyne nitens Tul. & 1 & 0,06 & 0,25 & 1,01 & 0,63 & 0,63 \\
\hline Amburana cearensis (Allemão) A.C.Sm. & 2 & 0,02 & 0,51 & 1,01 & 0,16 & 0,56 \\
\hline Mimosa arenosa (Willd.) Poir. & 1 & 0,01 & 0,25 & 1,01 & 0,14 & 0,47 \\
\hline Handroanthus impetiginosus (Mart. ex DC.) Mattos & 1 & 0,01 & 0,25 & 1,01 & 0,06 & 0,44 \\
\hline Genipa americana L. & 1 & 0,01 & 0,25 & 1,01 & 0,06 & 0,44 \\
\hline Dalbergia cearensis Ducke & 1 & 0,00 & 0,25 & 1,01 & 0,02 & 0,43 \\
\hline Total & 393 & 9,55 & 100 & 100 & 100 & 100 \\
\hline Nome Científico/Trecho Meio & NI & $\mathrm{AB}$ & DR & FR & DoR & VI \\
\hline Albizia inundata (Mart.) Barneby \& J.W.Grimes & 369 & 1,42 & 40,59 & 13,83 & 30,39 & 28,27 \\
\hline Chloroleucon foliolosum (Benth.) G.P.Lewis & 221 & 1,12 & 24,31 & 11,7 & 24,03 & 20,01 \\
\hline Celtis ehrenbergiana (Klotzsch) Liebm. & 92 & 0,70 & 10,12 & 11,7 & 15,08 & 12,3 \\
\hline Senegalia polyphylla (DC.) Britton \& Rose & 45 & 0,59 & 4,95 & 12,77 & 12,63 & 10,12 \\
\hline Geoffroea spinosa Jacq. & 66 & 0,42 & 7,26 & 11,7 & 8,89 & 9,28 \\
\hline Savia dictyocarpa Müll.Arg. & 77 & 0,21 & 8,47 & 12,77 & 4,5 & 8,58 \\
\hline Schinopsis brasiliensis Engl. & 21 & 0,09 & 2,31 & 11,7 & 1,97 & 5,33 \\
\hline Ziziphus joazeiro Mart. & 7 & 0,05 & 0,77 & 5,32 & 1 & 2,36 \\
\hline Triplaris gardneriana Wedd. & 4 & 0,03 & 0,44 & 2,13 & 0,73 & 1,1 \\
\hline Mimosa tenuiflora (Willd.) Poir. & 2 & 0,01 & 0,22 & 2,13 & 0,32 & 0,89 \\
\hline Eugenia florida DC. & 2 & 0,00 & 0,22 & 1,06 & 0,1 & 0,46 \\
\hline Senegalia langsdorffii (Benth.) Seigler \& Ebinger & 1 & 0,01 & 0,11 & 1,06 & 0,19 & 0,45 \\
\hline Coccoloba schwackeana Lindau & 1 & 0,00 & 0,11 & 1,06 & 0,09 & 0,42 \\
\hline Colubrina glandulosa Perkins & 1 & 0,00 & 0,11 & 1,06 & 0,08 & 0,42 \\
\hline Total & 909 & 4,67 & 100 & 100 & 100 & 100 \\
\hline
\end{tabular}

Revista Árvore, Viçosa-MG, v.37, n.6, p.1011-1023, 2013 
Tabela 2 - Cont

Table 2 - Cont

\begin{tabular}{lrrrrrrr} 
& Nome Científico/Trecho Lagoa da Prata & N & AB & DR & FR & DoR & D \\
Albizia inundata (Mart.) Barneby \& J.W.Grimes & 472 & 2,38 & 40 & 11,02 & 32,11 & 27,71 \\
Chloroleucon foliolosum (Benth.) G.P.Lewis & 345 & 2,01 & 29,24 & 11,02 & 27,1 & 22,45 \\
Celtis ehrenbergiana (Klotzsch) Liebm. & 82 & 0,51 & 6,95 & 9,32 & 6,91 & 7,73 \\
Schinopsis brasiliensis Engl. & 58 & 0,29 & 4,92 & 11,02 & 3,89 & 6,61 \\
Geoffroea spinosa Jacq. & 62 & 0,43 & 5,25 & 7,63 & 5,79 & 6,22 \\
Senegalia polyphylla (DC.) Britton \& Rose & 41 & 0,40 & 3,47 & 9,32 & 5,44 & 6,08 \\
Savia dictyocarpa Müll.Arg. & 24 & 0,07 & 2,03 & 8,47 & 0,91 & 3,8 \\
Mouriri pusa Gardner & 8 & 0,66 & 0,68 & 0,85 & 8,91 & 3,48 \\
Triplaris gardneriana Wedd. & 22 & 0,25 & 1,86 & 5,08 & 3,4 & 3,45 \\
Ziziphus joazeiro Mart. & 14 & 0,09 & 1,19 & 5,93 & 1,21 & 2,78 \\
Mimosa tenuiflora (Willd.) Poir. & 11 & 0,07 & 0,93 & 5,93 & 0,99 & 2,62 \\
Amburana cearensis (Allemão) A.C.Sm. & 18 & 0,05 & 1,53 & 4,24 & 0,74 & 2,17 \\
Coccoloba schwackeana Lindau & 12 & 0,12 & 1,02 & 2,54 & 1,64 & 1,73 \\
Colubrina glandulosa Perkins & 4 & 0,05 & 0,34 & 1,69 & 0,63 & 0,89 \\
Peltophorum dubium (Spreng.) Taub. & 2 & 0,01 & 0,17 & 1,69 & 0,11 & 0,66 \\
Annona vepretorum Mart. & 1 & 0,00 & 0,08 & 0,85 & 0,06 & 0,33 \\
Astronium fraxinifolium Schott & 1 & 0,00 & 0,08 & 0,85 & 0,06 & 0,33 \\
Genipa americana L. & 1 & 0,00 & 0,08 & 0,85 & 0,02 & 0,32 \\
Ximenia americana L. & 1 & 0,00 & 0,08 & 0,85 & 0,03 & 0,32 \\
Annona leptopetala (R.E.Fr.) H.Rainer & 1 & 0,00 & 0,08 & 0,85 & 0,04 & 0,32 \\
Total & 1180 & 7,42 & 100 & 100 & 100 & 100 \\
\hline
\end{tabular}

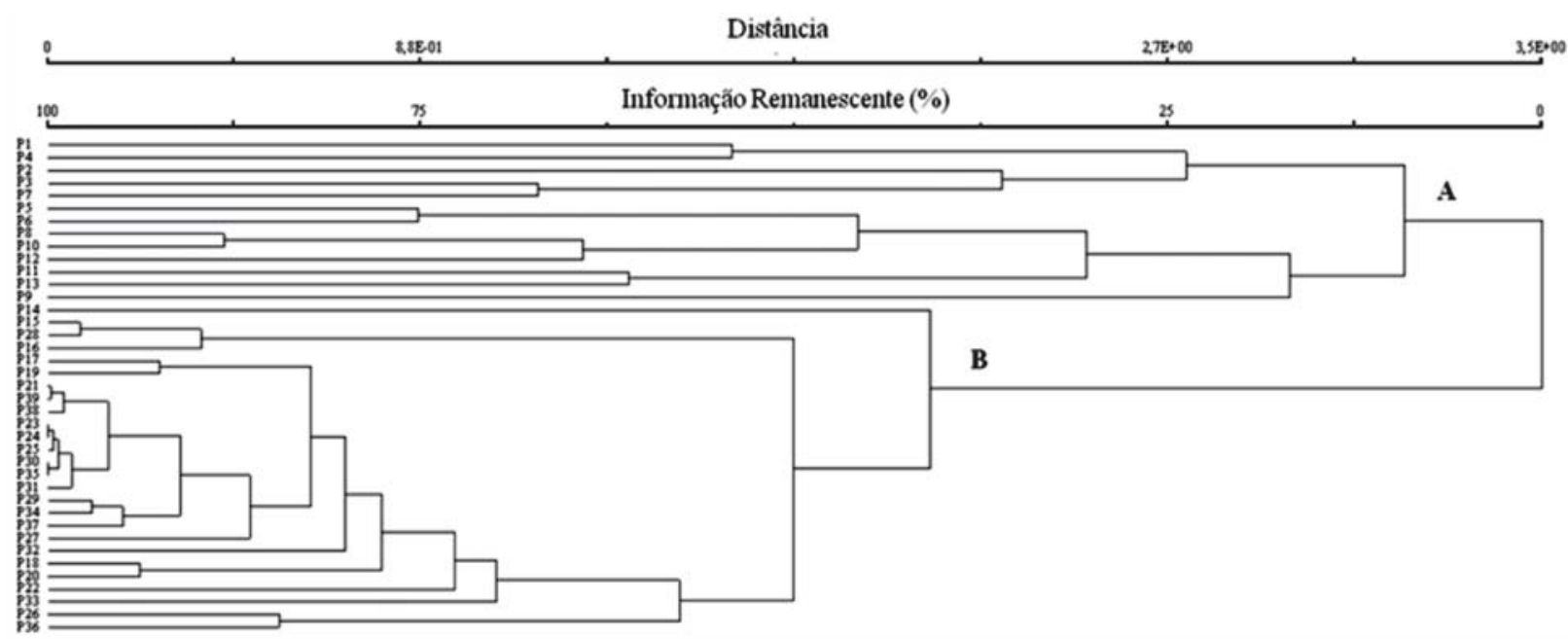

Figura 1 - Dendrograma obtido pelo método da ligação média (UPGMA) com base na Matriz de Similaridade de Jaccard, para dados de presença e ausência dos três trechos de Mata Ciliar estudados no Parque Estadual da Mata Seca, Norte de Minas Gerais (1-13 = São Francisco; 14-26 = Lagoa da Prata; e 27-39 = Meio).

Figure 1 - Dendrogram obtained by average linkage method (UPGMA) based on Jaccard similarity matrix for data of presence and absence of the three studied stretches of riparian vegetation in the Parque Estadual da Mata Seca, North of Minas Gerais.

altas, com maior área basal, enquanto o Meio e a Lagoa Prata mostraram maior densidade de indivíduos mais baixos e mais finos.
A dominância diferiu significativamente entre os trechos $(\mathrm{gl}=2 ; \mathrm{F}=6,683 ; \mathrm{p}<0,01)$, todavia o teste de Tukey mostrou que a Lagoa da Prata não se diferenciou 
Tabela 3 - Correlação de Pearson para os valores de área basal e densidade de indivíduos com as variáveis ambientais analisadas no gradiente de floresta ciliar inundável no PEMS. R - = coeficiente de correlação de Pearson; e $\mathrm{R}^{2}$ = regressão linear.

Table 3 - Pearson's correlation for the values of basal area and density of individuals with environmental variables analyzed in the gradient of riparian wetland in the PEMS. $R$-= Pearson correlation coefficient; and $R^{2}=$ linear regression.

\begin{tabular}{|c|c|c|c|c|c|c|}
\hline \multirow{2}{*}{ Variáveis do solo } & \multicolumn{3}{|c|}{ Área Basal Total } & \multicolumn{3}{|c|}{ Densidade } \\
\hline & $\mathrm{R}$ & $\mathrm{R}^{2}$ & $\mathrm{p}$ & $\mathrm{R}$ & $\mathrm{R}^{2}$ & $\mathrm{P}$ \\
\hline Umidade & -0.015 & 0.0002 & ns & 0.627 & 0.393 & $* * *$ \\
\hline $\mathrm{pH}$ em $\mathrm{H}_{2} \mathrm{O}$ & 0.398 & 0.1584 & $*$ & -0.71 & 0.51 & $* * *$ \\
\hline $\mathrm{P}\left(\mathrm{mg} \cdot \mathrm{dm}^{-3}\right)$ & 0.516 & 0.2663 & $* *$ & -0.59 & 0.35 & $* * *$ \\
\hline $\mathrm{K}^{+}\left(\mathrm{mg} \cdot \mathrm{dm}^{-3}\right)$ & 0.493 & 0.243 & $* *$ & -0.1 & 0.01 & Ns \\
\hline $\mathrm{Ca}^{++}\left(\mathrm{mg} \cdot \mathrm{dm}^{-3}\right)$ & 0.024 & 0.0006 & ns & 0.361 & 0.13 & $*$ \\
\hline $\mathrm{Mg}^{++}\left(\mathrm{mg} \cdot \mathrm{dm}^{-3}\right)$ & -0.324 & 0.105 & $*$ & 0.606 & 0.367 & $* * *$ \\
\hline $\mathrm{Al}^{+++}\left(\mathrm{mg} \cdot \mathrm{dm}^{-3}\right)$ & -0.352 & 0.1239 & $*$ & 0.44 & 0.194 & $* *$ \\
\hline MO (dag.kg $\left.{ }^{-1}\right)$ & -0.25 & 0.0625 & ns & 0.66 & 0.436 & $* * *$ \\
\hline $\mathrm{Zn}\left(\mathrm{mg} \cdot \mathrm{dm}^{-3}\right)$ & 0.494 & 0.244 & $* *$ & -0.59 & 0.342 & $* * *$ \\
\hline Fe $\left(\mathrm{mg} . \mathrm{dm}^{-3}\right)$ & -0.172 & 0.0296 & ns & 0.317 & 0.10 & $*$ \\
\hline Mn (mg.dm $\left.{ }^{-3}\right)$ & 0.48 & 0.2304 & $* *$ & -0.56 & 0.315 & $* * *$ \\
\hline $\mathrm{Cu}\left(\mathrm{mg} \cdot \mathrm{dm}^{-3}\right)$ & -0.126 & 0.0159 & ns & 0.313 & 0.098 & Ns \\
\hline Areia grossa (\%) & 0.038 & 0.0014 & ns & -0.1 & 0.011 & Ns \\
\hline Areia fina (\%) & 0.376 & 0.1414 & $*$ & -0.6 & 0.365 & $* * *$ \\
\hline Silte (\%) & -0.322 & 0.1037 & $*$ & 0.44 & 0.194 & $* *$ \\
\hline Argila (\%) & -0.382 & 0.1459 & $*$ & 0.673 & 0.453 & $* * *$ \\
\hline
\end{tabular}

$* \mathrm{P}<0,05 ; * * \mathrm{P}<0,01 ; * * * \mathrm{p}<0,001 ;$ e ns = não significativo.

das outras duas comunidades. Celtis ehrenbergiana deteve 55,44\% da dominância do São Francisco, enquanto a Albizia inundata obteve a maior dominância para o Meio (30,39\%) e Lagoa da Prata (32,11\%) (Tabela 2).

As três espécies com maiores valores de importância (VI) nos trechos Meio e Lagoa da Prata foram iguais, sendo elas: A. inundata, Chloroleucon foliolosum e Celtis ehrenbergiana (Tabela 2). Entretanto, nesses trechos houve alteração nas espécies em comparação com o São Francisco, no qual as espécies com maiores VI foram C. ehrenbergiana, seguido pelo Inga vera e Ziziphus joazeiro (Tabela 3). A. inundata, espécie com maior VI neste estudo, exibiu a maior densidade e frequência, estando presente em todas as unidades amostrais do Meio e da lagoa da Prata e 69\% das parcelas do São Francisco.

A área basal apresentou correlações positivas e significativas com $\mathrm{pH}, \mathrm{P}, \mathrm{K}, \mathrm{Zn}, \mathrm{Mn}$ e areia fina, bem como correlações negativas e significativas com Mg, Al, silte e argila (Tabela 3). Entretanto, a densidade de indivíduos apresentou correlações significativas com a maior parte das variáveis analisadas, exceto com $\mathrm{K}, \mathrm{Cu}$ e areia grossa. Os valores de umidade, Ca, Mg, Al, matéria orgânica, Fe, silte e argila apresentaram correlações positivas, enquanto $\mathrm{pH}, \mathrm{P}, \mathrm{Zn}, \mathrm{Mn}$ e areia fina exibiram correlações negativas (Tabela 3) com a densidade de indivíduos.

\section{DISCUSSÃO}

\subsection{Variáveis edáficas}

O fragmento estudado apresenta diferentes condições edáficas, com solos variando sua textura de franco-arenosa a argilosa, que propiciam o estabelecimento de espécies vegetais adaptadas a cada microambiente formado. As parcelas com alto teor de partículas finas apresentam dificuldade na infiltração de água, colaborando para a formação de solos saturados, muitas vezes lamacentos, encharcados e mais compactados (RIZZINI, 1997), como os encontrados nos trechos Lagoa da Prata e Meio. Com a redução de oxigênio nesses solos, não há decomposição suficiente da matéria orgânica, ocorrendo seu acúmulo e ocasionando o aumento da acidez, que está associado com o elevado teor de alumínio (SILVA et al., 2006). Ao contrário, os solos do trecho São Francisco, mais arenosos em comparação com os outros dois trechos, possuem maior eficiência da infiltração da água, não proporcionando sua retenção nem o acúmulo de matéria orgânica (SILVA et al., 2009).

Revista Árvore, Viçosa-MG, v.37, n.6, p.1011-1023, 2013 

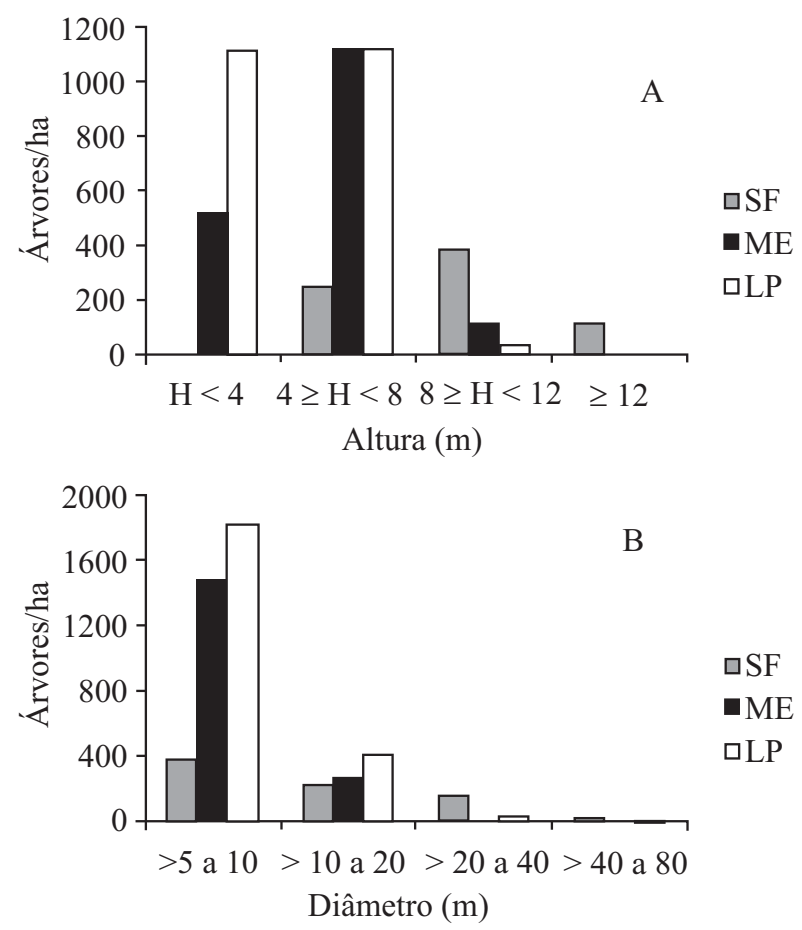

Figura 2 - Distribuição da densidade de indivíduos arbóreos $\geq 5 \mathrm{~cm}$ de DAP em classes de altura (A) e diâmetro (B) em três trechos de Floresta Ciliar inundável, no PEMS, MG.

Figure 2-Density distribution of the tree and shrub individuals $\geq 5 \mathrm{~cm} D B H$ in height classes (A) and diameter (B) in three sections of flooded riparian forest of the PEMS, MG.

O gradiente florestal apareceu regido pela umidade e pelo $\mathrm{pH}$ do solo que foram associados a uma série de propriedades químicas (como o P, Mg, MO, Zn e $\mathrm{Mn}$ ). Esse resultado reflete os resultados de muitos outros estudos sobre vegetação ciliar com foco não só em áreas úmidas (BARDDAL et al., 2005; PINTO et al., 2005), mas também em áreas bem drenadas (PINTO et al., 2005; CAMARGOS et al., 2008). Considerando os critérios da análise realizada, o solo localizado no trecho São Francisco não apresentou teores de alumínio, além de possuir valores de $\mathrm{pH}$ tendendo à neutralidade. Os teores de $\mathrm{P}$, Zn e Mn foram maiores nesse trecho do que nas outras duas áreas. A Lagoa da Prata e o Meio possuíram solos com acidez média, altos teores de Fe e baixo teor de fósforo e demais nutrientes. Assim, a variação química do solo entre os três trechos está relacionada, de certa forma, com o gradiente florestal, textura e umidade do solo. Vale salientar que a interpretação da análise química do solo foi estabelecida de acordo com Alvarez et al. (1999).

\subsection{Variações na diversidade e estrutura das comunidades}

O número de espécies registrado no gradiente amostrado é considerado baixo no contexto das florestas ciliares, já que Rodrigues e Nave (2004) listraram um total de 955 espécies arbustivo-arbóreas compiladas de 43 levantamentos florísticos e fitossociológicos de florestas ciliares do Brasil extra-amazônico. Entretanto, quando comparado com as florestas inundáveis, os índices de diversidade de Shannon apresentam valores dentro do esperado, corroborando outros estudos realizados nesse tipo de ambiente (BRITO et al., 2006; CAMARGOS et al., 2008), que se referem a florestas inundáveis como sistemas de baixa diversidade. Esse resultado confirma ainda informações de que áreas sujeitas a inundações periódicas ou esporádicas possuem menor diversidade que florestas ciliares sem alagamento (JOLY, 1991). Essa situação, provavelmente, é efeito do estresse imposto no solo pela saturação hídrica, no qual se estabelecem apenas as espécies que toleram tal condição (LOBO; JOLY, 2004), e comumente algumas dessas espécies se tornam dominantes (BARDDAL et al., 2004).

O baixo valor da equabilidade encontrado neste fragmento reflete a dominância das espécies Albizia inundata, Chloroleucon foliolosum e Celtis ehrenbergiana, que juntas totalizaram $74 \%$ dos indivíduos amostrados. A alta dominância dessas espécies pode estar relacionada com sua categoria sucessional, na qual Albizia inundata é considerada pioneira e Celtis ehrenbergiana é tida como secundária inicial (LORENZI, 1992; SARAIVA, 2011). Além disso, a dominância de poucas espécies é um indicativo que a área possui estresse, podendo se destacar a alta saturação hídrica do solo e o clima severo atuante no polígono das secas.

A diferença do número de indivíduos nos trechos amostrados sugere um gradiente de densidade decrescente da Lagoa da Prata para o São Francisco, relacionados com a textura e variáveis químicas do solo. Comparando com o valor de densidade total encontrado no fragmento, o valor foi maior que os obtidos nas florestas ciliares não inundáveis (BATTILANI et al., 2005) e semelhantes a florestas que sofrem alagamentos (BIANCHINI et al., 2003; BRITO et al., 2006; CAMARGOS et al., 2008; MARTINS et al., 2008; MAGNAGO et al., 2010).

Revista Árvore, Viçosa-MG, v.37, n.6, p.1011-1023, 2013 
Os trechos da Lagoa da Prata e do Meio apresentaram maior densidade de indivíduos com menor altura em relação ao encontrado no São Francisco. O alagamento mais pronunciado nesses dois primeiros trechos é um dos fatores prováveis para esse resultado, já que áreas que apresentam saturação hídrica normalmente propiciam menor crescimento em altura que áreas mais drenadas (BARDDAL et al., 2004). Nessas condições, a escassez de oxigênio para a respiração e redução da condutividade hidráulica do sistema radicular dificulta o metabolismo das espécies vegetais (JOLY, 1991), promovendo o baixo desenvolvimento estrutural da vegetação nessas condições. A instabilidade do solo durante os períodos de inundação e a pequena profundidade do sistema de raízes em razão da superficialidade do lençol freático são características que ampliam a possibilidade de tombamento dos indivíduos mais altos, limitando o crescimento em altura de outros (BIANCHINI et al., 2003).

A área basal varia com as condições de solo, água e luz (SCHAAF et al., 2006). Assim, a grande concentração de indivíduos nas classes inferiores de diâmetro pode ser decorrente da baixa disponibilidade de nutrientes e altos valores de umidade do solo, os quais podem restringir o desenvolvimento dos indivíduos. O formato da curva exponencial negativa apresentado pela distribuição de diâmetros é um padrão considerado comum em florestas tropicais (FELFILI et al., 1997), sendo consequência da dinâmica florestal, já que existe relação inversa entre número e tamanho (porte) dos indivíduos amostrados (OLIVEIRA et al., 2001). Schaaf et al. (2006) concluíram que a distribuição diamétrica reflete as características autoecológicas das espécies. Assim, possuir grande número de indivíduos de grande porte nem sempre é indicativo de que a espécie irá se manter na comunidade, mas sim sua capacidade de competir dentro do seu nicho ecológico.

A Albizia inundata, espécie com maior densidade no gradiente amostrado, também superou as outras espécies em frequência, sendo também encontrada nas florestas ciliares de países da América Latina, incluindo o domínio da Caatinga (ROCHA et al., 2004). A baixa frequência da maioria das espécies pode ser reflexo de uma distribuição preferencial em relação aos diferentes hábitats amostrados no gradiente. Como exemplo, podem-se citar os extremos das espécies Guarea guidonia e Celtis ehrenbergiana. A primeira foi representada por sete indivíduos em uma única parcela e a segunda, por 385 indivíduos em 35 parcelas, o que retrata o contraste fitossociológico das espécies nesse ambiente.

\section{CONCLUSÃO}

A Floresta Ciliar do PECG possui densidade e área basal fortemente influenciadas pela umidade, $\mathrm{pH}, \mathrm{P}$, Mg, Al, matéria orgânica, Zn, Mn, areia fina e argila. Assim, pôde-se constatar que a estrutura dos três trechos estudados está correlacionada com os fatores texturais, químicos e umidade do solo, ocasionando variações fitossociológicas nas comunidades estudadas.

\section{AGRADECIMENTOS}

À Fapemig, pelo financiamento do Projeto (Edital Universal 01/2009 - Processo APQ-02097-09); à Capes, pela bolsa de doutorado da primeira autora; e ao CNPq, pela bolsa de produtividade em pesquisa ao segundo autor; e ao IEF, pelo apoio logístico.

\section{REFERÊNCIAS}

ALVAREZ V., V. H.et al.Interpretação dos resultados das análises de solos. In: RIBEIRO, A. C.; GUIMARÃES, P. T. G.; ALVAREZ V., V. H. (Ed.). Recomendações para o uso de corretivos e fertilizantes em Minas Gerais $-5^{a}$ aproximação. Viçosa, MG: Comissão de fertilidade do Solo em Minas Gerais, 1999. p.25-32.

APG III - THE ANGIOSPERM PHYLOGENY GROUP. An update of the Angiosperm Phylogeny Group classification for the orders and families of flowering plants: APG III.

Botanical Journal of the Linnean Society, v.161, n.20, p.105-121, 2009.

BAILLIE, I. C.; ASHTON, P. S. Some soil aspects of the nutrient cycle in mixed dipterocarp forests in Sarawak. In: SUTTON, S. L.; WHITMORE, T. C.; CHADWICK, A. C. (Ed.). Tropical rain forest: ecology and management. Oxford: Blackwell Scientific Publication, 1983. p.239-251.

BARDDAL, M. L. et al. Fitossociologia do subbosque de uma Floresta Ombrófila Mista Aluvial, no município de Araucária, PR. Ciência

Florestal, v.14, n.1, p.35-45, 2004. 
BATTILANI, J. L.; SCREMIN-DIAS, E.; SOUZA, A. L. Fitossociologia de um trecho da mata ciliar do rio da Prata, Jardim, MS, Brasil. Acta BotanicaBrasilica, v.19, n.3, p.597-608, 2005.

BIANCHINI, E. et al. Diversidade e estrutura de espécies arbóreas em área alagável do município de Londrina, Sul do Brasil. Acta

BotanicaBrasilica, v.17, n.3, p.405-419, 2003.

BRITO, E. R. et al. Estrutura fitossociológica de um fragmento natural de floresta inundável em área de orizicultura irrigada, município de Lagoa da Confusão, Tocantins. RevistaÁrvore, v.30, n.5, p.829-836, 2006.

BROWER, J. E.; ZAR, J. H. Field and laboratory methods for general ecology. Dubuque: W.M.C. Brow, 1984. 226p.

CAMARGOS, V.L. et al. Influência de fatores edáficos sobre variações florísticas na FlorestaEstacional Semidecídua no entorno da Lagoa Carioca, Parque Estadual do Rio Doce, MG, Brasil. Acta Botanica Brasílica, v.22, n.1, p.75-84, 2008.

CIENTEC. Mata Nativa 2: manual do usuário. Viçosa, MG: Cientec, 2006. 295p.

DÉSILETS, P.; HOULE, G. Effects of resource availability and heterogeneity on the slope of the species-area curve along a floodplain-upland gradient. Journal of Vegetation Science, v.16, n.5, p.487-496, 2005.

DRUMMOND, G. M. et al. Biodiversidade em Minas Gerais: um atlas para sua conservação. 2.ed. Belo Horizonte:Fundação Biodiversitas, 2005. 222p.

EMPRESA BRASILEIRA DE PESQUISA AGROPECUÁRIA - EMBRAPA. Manual de Métodos de análise de solo. 2.ed. Rio de Janeiro: 1997. 212p.

FELFILI, J. M. et al. Comparação florística e fitossociológica do cerrado nas Chapadas Pratinha e dos Veadeiros. In: LEITE, L. L.; SAITO, C. H. (Ed.). Contribuição ao conhecimento ecológico do Cerrado. Brasília: Universidade de Brasília, 1997. p.6-11.
GILBERT, N. Statistics. Philadelphia: W.B. SaundersCompany, 1976. 364p.

HUBBELL, S. P.; FOSTER, R. B. Diversity of canopy trees in a neotropical forest and implications for conservation. In: SUTTON, S. L.; WHITMORE, T. C.; CHADWICK, A. C. (Ed.). Tropical rain forest: ecology and management. Oxford: Blackwell Scientific Publication, 1983. p.25-41.

HUSTON, M. A. Soil nutrients and the tree species richness in Costa Rican forests.

Journal Biogeography, v.7, p.147-157, 1980.

INSTITUTO ESTADUAL DE FLORESTAS- IEF. Parecer técnico para a criação do Parque Estadual da Mata Seca. Relatório técnico. Belo Horizonte: 2000.

JACOMINE, P. K. T. Solos sob matas ciliares. In: RODRIGUES, R. R.; LEITÃO FILHO, H. F. (Ed.). Matas Ciliares: conservação e recuperação. São Paulo: EDUSP/FAPESP, 2004. p.27-31.

JOLY, C. A. Flooding tolerance in tropical trees. In: JACKSON, M. B.; DAVIS, D.D.; LAMBERS, H. (Ed.). Plant under oxygen deprivation. The Hague: SPB Academic Publishing, 1991. p.23-34.

KÖPPEN, W. Climatologia: con un estudio de los climas de la tierra. México: Fondo de Cultura Econômica, 1948. 478p.

LOBO, P.C.; JOLY, C.A. Aspectos ecofisiológicos da vegetação de mata ciliar do sudeste do Brasil. In: RODRIGUES, R. R.; LEITÃO FILHO, H. F. (Ed.). Matas Ciliares: conservação e recuperação. São Paulo: EDUSP/FAPESP, 2004. p.143-157.

LORENZI, H. Árvores Brasileiras: manual de identificação e cultivo de plantas arbóreas nativas do Brasil. 2.ed. Nova Odessa: Plantarum, 2002. v.2. 368p.

MADEIRA, B. G. et al. Changes in tree and liana communities along a successional gradient in a tropical dry forest in southeastern Brazil. Plant Ecology, v.201, n.1, p.291-304, 2009.

Revista Árvore, Viçosa-MG, v.37, n.6, p.1011-1023, 2013 
MAGNAGO, L.F.S. et al.Gradiente fitofisionômicoedáfico em formações florestais de Restinga no sudeste do Brasil. Acta Botânica Brasílica, v.24, n.3, p.734-746, 2010.

MAGURRAN, A. E. Ecological diversity and its measurement. New Jersey: Princeton University Press, 1988. 192p.

MARTINS, S. V. Recuperação de matas ciliares. 2.ed. Viçosa, MG: Aprenda Fácil, 2007. 255p.

MARTINS, S.V. et al. Distribuição de espécies arbóreas em um gradiente topográfico de Floresta Estacional Semidecidual em Viçosa, MG.

ScientiaForestalis, n.641, p.72-181, 2003.

MARTINS, S.V. et al. Floristic composition of two wetland forests in Araguaian Plain, State of Tocantins, Brazil, and comparison with other areas. Revista Árvore, v.32, n.1, p.129-141, 2008.

MCCUNE, B.; MEFFORD, M. J. PC-ORD: Multivariate analysis of ecological data. Version 5.10. Oregon: Gleneden Beach, 2006. 126p.

MELLO, J. M.; SCOLFORO, J. R.S.; CARVALHO, L. M. T.(Ed.). Inventário Florestal de Minas Gerais: Floresta estacional decidual - Florística, estrutura, diversidade, similaridade, distribuição diamétrica e de altura, volumetria, tendências de crescimento e manejo florestal. Lavras:

Universidade Federal de Lavras, 2008. 265p.

MUELLER-DOMBOIS, D.; ELLENBERG. H. Aims and methods of vegetation ecology. 5.ed. New York: John Wiley \& Sons, 1975. 570p.

OLIVEIRA-FILHO, A. T. et al. Variation in tree community composition and structure with changes in soil properties within a fragment of semideciduous forest in south-eastern Brazil. Edinburgh Journal of Botany, v.58, n.1, p.139-158, 2001.

PINTO, L. V. A.et al. Distribuição das espécies arbóreo-arbustiva ao longo do gradiente de umidade do solo de nascentes pontuais da bacia hidrográfica do Ribeirão Santa Cruz, Lavras, MG. Cerne, v.11, n.3, p.294-305, 2005.
PINTO, S.I.C. et al. Influence of environmental variables on the shrub and tree species distribution in two Semideciduous Forest sites in Viçosa, Minas Gerais, Brazil. International Journal of Tropical Biology, v.56, n.3, p.1557-1569, 2008.

RIZZINI, C. T. Tratado de fitogeografia do Brasil: aspectos ecológicos, sociológicos e florísticos. Rio de Janeiro: Âmbito Cultural Edições, 1997. 747p.

ROCHA, P. L. B.; QUEIROZ, L. P.; PIRANI, J. R. Plant species and habitat structure in a sand dune field in the Brazilian Caatinga: a homogeneous habitat harboring an endemic biota. Revista Brasileira de Botânica, v.27, n.4, p.739-755, 2004.

RODRIGUES, R. R.; NAVE, A. G. Heterogeneidade florística das matas ciliares. In: RODRIGUES, R.R.; LEITÃO FILHO, H.F. (Ed.) Matas ciliares: conservação e recuperação. São Paulo: Universidade de São Paulo, 2000.p.45-71

RUGGIERO, P. G. C.et al.Soil-vegetation relationships in cerrado (Brazilian savanna) and semideciduous forest, Southeastern Brazil. PlantEcology, v.160, p.1-16, 2002.

SARAIVA, D. D. Composição e estrutura de uma floresta ribeirinha no sul do Brasil. Biotemas, v.20, n.4, p.49-58, 2011.

SCHAAF, L. B. et al. Alteração na estrutura diamétrica de uma Floresta Ombrófila Mista no período entre 1979 e 2000. Revista Árvore, v.30, n.2, p.283-295, 2006.

SCHAEFER, C. E. G. R. et al. Relações solovegetação em alguns ambientes brasileiros: fatores edáficos e florística. In: MARTINS, S.V. (Ed.). Ecologia de florestas tropicais. Viçosa, MG: Universidade Federal de Viçosa, 2009. p.143-184.

SILVA, L. S.; CAMARGO, F. A. O.; CERETTA, C. A. Composição da fase sólida orgânica do solo. In: MEURER, E. J. (Ed.). Fundamentos de química do solo. Porto Alegre: Evangraf, 2006. p.63-90.

SILVA, A.C. et al. Comparação florística de florestas inundáveis das regiões Sudeste e Sul do Brasil. Revista Brasileira de Botânica, v.30, n.2, p.257-269, 2007. 
SILVA, A.C. et al. Florística e estrutura da comunidade arbórea em fragmentos de floresta aluvial em São Sebastião da Bela Vista, Minas Gerais, Brasil. Revista Brasileira de Botânica, v.32, n.2, p.283-297, 2009.

STATSOFT INC. Statistica: data analysis software system. Version 7.0. Tulsa: Statsoft, 2004.

VALENTIM, J. L. Ecologia numérica: uma introdução à análise multivariada de dados. Rio de Janeiro: Interciências, 2000. 118p.
WHITMORE, T. C. Change with time and the role of cyclones in tropical rain forest of Kolombangara Solomon Islands. Oxford: Commonwealth Forest Institute, 1974. 78p.

WITTMANN, F.; JUNK, W. J.; PIEDADE, M. T. F. The varzea forests in Amazonia: flooding and the highly dynamic geomorphology interact with natural forest succession. Forest Ecology and Management, v.196, n.2/3, p.199-212, 2004.

ZAR, J.H. Biostatistical analysis. 4.ed. New Jersey: Prentice Hall, 1998. 929p. 
